\title{
Solar Magnetic Loops Observed with TRACE and EIT
}

\author{
Markus J. Aschwanden \& Alan M. Title \\ Lockheed Martin, Solar 83 Astrophysics Laboratory, \\ Palo Alto, CA 94304, USA
}

\begin{abstract}
We select some highlights and new results that have been obtained from detailed "microscopic" observations of coronal loop structures with the Transition Region and Coronal Explorer (TRACE) and Extreme Ultraviolet Imager (EIT) instruments, including: (1) the inhomogeneous substructure of EUV loops, (2) the dynamic and non-hydrostatic nature, (3) the non-uniform heating, (4) the magnetic topology at the loop footpoints, (5) the magnetic energy budget for heating, and (6) oscillations and waves in coronal loops.
\end{abstract}

\section{Introduction}

TRACE has provided us a "microscope" that allows us to dissect the anatomy of the solar corona in unprecedented detail. It is not only the high angular resolution (0.5" pixel size, corresponding to $350 \mathrm{~km}$ on the solar surface) that clarifies the details of the myriads of coronal fine structure and enhances their contrast, but equally important is also the temperature discrimination of narrow-band filters that helps us to disentangle the multi-temperature corona, as well as the faster cadence that reveals us the dynamic evolution. Thanks to these improved capabilities in EUV we discovered new dynamic phenomena, for example MHD oscillations of coronal loops, which could not be imaged previously because of insufficient resolution, contrast, and cadence. The combination of higher spatial resolution and better temperature discrimination allows us also to isolate "monolithic" coronal loops from the multi-thermal background, and to examine their pristine density and temperature structure unconfused by the ambient plasma, a crucial prerequisite to measure uncontaminated physical quantities and to examine the physical momentum and energy balance of coronal structures. Only by means of these capabilities we can hope to arrive at a sound understanding of the fundamental physical plasma processes, which provide us the tools to model also stellar atmospheres with more realistic models. Such more reliable measurements of uncontaminated physical parameters will lead to realistic atmospheric models, which can be validated by scaling them to stars with different masses, temperatures, and magnetic field strengths. 

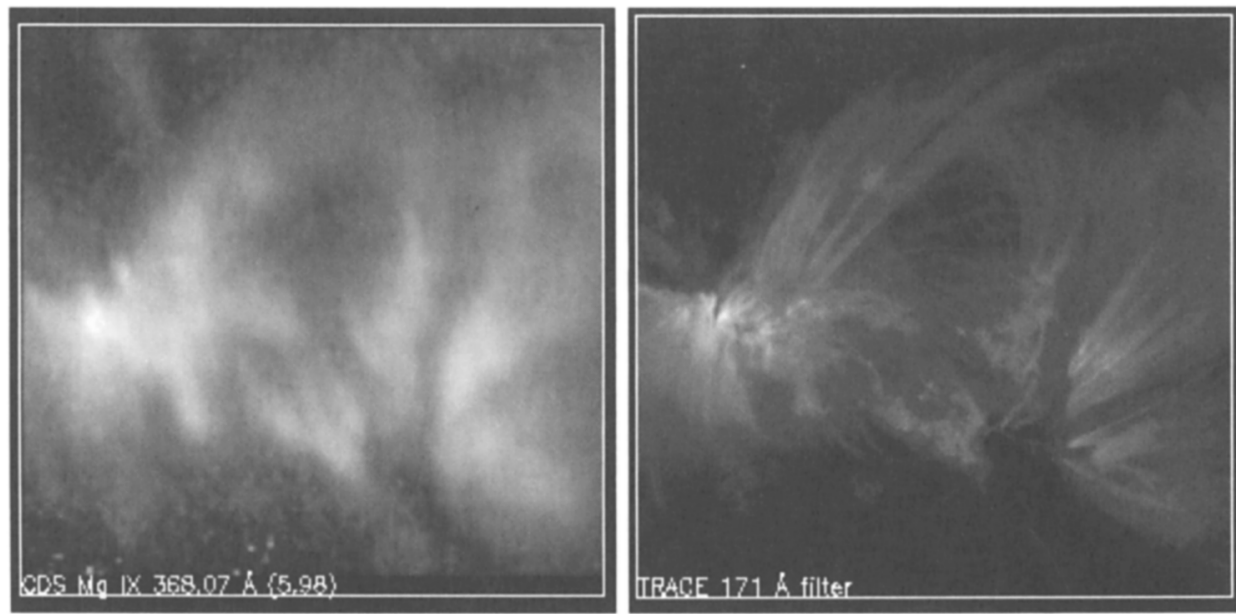

Figure 1. Cospatial and contemporaneous observations of a coronal loop system by SoHO/CDS (Mg IX, 368.07 $\AA, \log (\mathrm{T})=5.98)$ and TRACE (Fe IX/X, $171 \AA, \log (\mathrm{T})=6.0)$. Both images are taken in an identical temperature range. Note that the loop system that is perceived as a single loop with CDS consists of at least a dozen loop strands in the TRACE image.

\section{Highlights and New Results}

\subsection{Inhomogeneous Substructure of EUV Loops}

Interestingly, the most "crispy" images of the solar corona are generally obtained at a wavelength of $171 \AA$, in the Fe IX/X lines with a formation temperature of $T_{e} \approx 1.0 \mathrm{MK}$, where the differential emission measure (DEM) distribution shows a peak for the quiet corona. An example is shown in Fig. 1 (right frame), which conspicuously illustrates the inhomogeneous structure of closely-packed loop strands (like "spaghettis") that are perceived as a single loop structure with other instruments with poorer spatial resolution (e.g., with CDS, which has an effective resolution of $\approx 10 "-15 "$; Fig. 1 left frame). Given the narrowband response of the TRACE $171 \AA$ filter, all loop strands have a temperature in the narrow range of $T_{e} \approx 0.8-1.2 \mathrm{MK}$. Contemporaneous images recorded in different temperature ranges show that the brightest structures are not exactly co-spatial in different (non-overlapping) temperature bands, which implies that each structure has a relatively narrow temperature range (in cross-section and along their lengths) at a given time of their evolution (Schrijver et al. 1999; Aschwanden et al. 2000a). This aspect spawned some controversy, because TRACE has narrow-band temperature filters and SoHO/CDS always observes a broad DEM distribution for what appears to be a single loop in CDS (Schmelz et al. 2001; Martens et al. 2002). However, it has been demonstrated that the broad DEM inferred from SoHO/CDS consists of multiple, narrow temperature peaks, which indicate multiple, near-isothermal loop structures along the same line-of-sight that cannot be resolved with the poorer spatial resolution of CDS 
The Non-Hydrostatic Sun



The Hydrostatic Sun

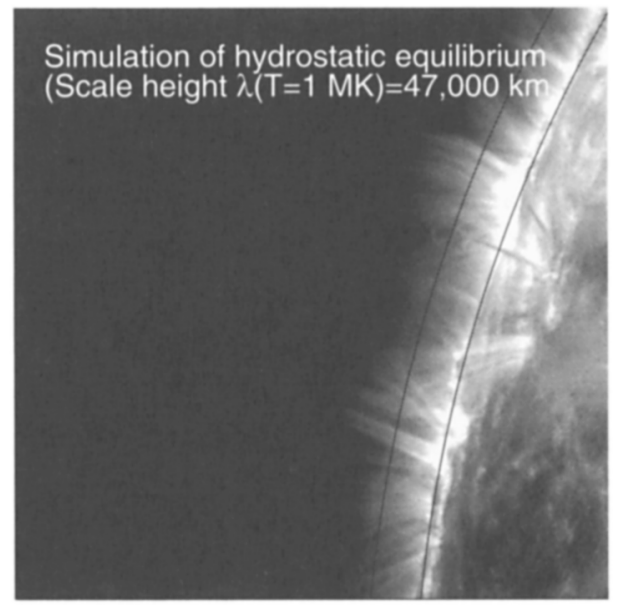

Figure 2. An active region with many loops that have an extended scale height of $\lambda_{p} / \lambda_{T} \lesssim 3-4$ (left panel) has been scaled to the hydrostatic thermal scale height of $T=1 \mathrm{MK}$ (right panel). The pressure scale height of the $1 \mathrm{MK}$ plasma is $\lambda_{T}=46 \mathrm{Mm}$, but the observed flux is proportional to the emission measure $\left(F \propto E M \propto n_{e}^{2}\right)$, which has the half pressure scale height $\lambda_{T} / 2=23 \mathrm{Mm}$ (indicated with a circle above the limb in right frame.

(Aschwanden 2002a). Thus, the coronal EUV loops seem to be inhomogeneous in density and temperature down to $\approx 1$ ", where elementary loop strands seem to be resolved with TRACE and reveal a monolithic temperature and density. Obviously this upper limit of $\approx 1$ " tells us some fundamental limit on the homogeneity of the highly localized heating function, which incidentally coincides with the spatial scale of the photospheric granulation.

\subsection{Non-Hydrostatic Loops}

Traditional models of solar and stellar atmospheres generally assume a hydrostatic structure with gravitational stratification. This concept can also be applied to a highly inhomogeneous corona, where myriads of loop structures coexist, each one representing an isolated "mini-atmosphere" with its own pressure scale height depending on its temperature. Since the quiet corona has a DEM with a broad peak in the temperature range of $T_{e} \approx 1-3 \mathrm{MK}$, we expect a mixture of hydrostatic scale heights in the range of $\lambda_{T} \approx 50-150 \mathrm{Mm}$. This general hydrostatic structure has been confirmed in many quiet-Sun regions from SoHO/EIT (Aschwanden et al. 2000a), Yohkoh (Aschwanden \& Acton 2001), and TRACE data. In active regions, however, TRACE observations clearly show that many coronal loops are not in hydrostatic equilibrium, but rather have density and pressure scale heights $\lambda_{p}$ in excess of their thermal scale height $\lambda_{T}$ by factors of up to $\lambda_{p} / \lambda_{T} \lesssim 3-4$ (Aschwanden, Nightingale, \& Alexander 2000b; Lenz et al. 1999). An example of such extremely non-hydrostatic loops is shown 


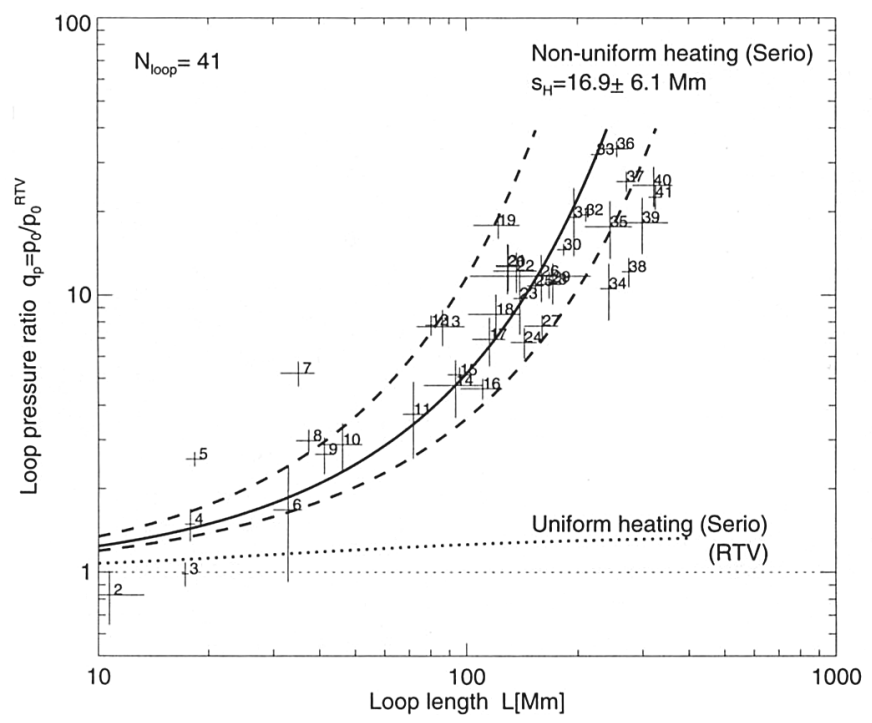

Figure 3. A fit of the non-uniform heating model $\left(R T V S_{p h}\right)$ of Serio et al. (1981), to the observed loop pressure factors $q_{p}=p_{0} / p_{0}^{R T V}$ is shown The best fit yields a heating scale height of $s_{H}=16.9 \pm 6.1$ $\mathrm{Mm}$ : the mean is shown with a thick solid line, the $\pm \sigma_{s_{H}}$ standard deviations with dashed lines. Note that the alternative model with uniform heating (dotted line) of Serio et al. (1981), $R T V S_{p}$, or Rosner et al. (1978), $R T V$, is not consistent with the data.

in Fig. 2 (left frame), while the expected hydrostatic template is shown in Fig. 2 (right frame). A sample of 41 coronal loops has been modeled with hydrostatic solutions of their temperature $T_{e}(s)$ and density profiles $n_{e}(s)$ along their length $s$ in the 171 and $195 \AA$ channels, using hydrostatic solutions over a large parameter space of heating functions, but two thirds of this sample could not be fitted by any hydrostatic solution (Aschwanden, Schrijver, \& Alexander 2001). This implies that most loops studied with TRACE reveal a dynamic character and large deviations from hydrostatic equilibrium. It was noted that many of these active region loops appear first in the hotter TRACE passbands (284 $\AA, 195 \AA$ ), before they appear in the cooler TRACE passband (171 $\AA$ ), which was interpreted as evidence for cooling from $T_{e} \approx 2.0 \mathrm{MK}$ down to $T_{e} \approx 1.0$ MK (Winebarger, Warren, \& Seaton 2003). However, the time delay of their appearance between hotter and cooler passbands was found to be inconsistent with the simplest hydrodynamic model of a heated loop that cools by conductive and radiative cooling (Warren, Winebarger, \& Hamilton 2002), leading to the conclusion that more complex models are needed with either temporally intermittent heating functions or spatially filamented loop strands. All these observational results emphasize the spatial inhomogeneity of EUV loops. 


\subsection{Non-Uniform Heating}

Another long-standing paradigm was the assumption of uniform heating of coronal structures, leading to the broad acceptance of the RTV scaling laws (Rosner, Tucker, \& Vaiana 1978), which are also widely used in modeling of stellar atmospheres. The derivation of the RTV scaling laws makes two fundamental assumptions, (1) that the pressure is constant along the coronal loops, and (2) that the heating rate is uniformly distributed along the loops. The RTV scaling laws have been applied mostly to soft X-ray loops, which are hotter $T_{e} \approx 2-8$ $\mathrm{MK})$ than EUV loops $\left(T_{e} \approx 1-2 \mathrm{MK}\right)$, and thus the approximation of a constant pressure works better for soft X-ray loops, which probably explains why deviations from the RTV law were not noticed earlier. The RTV scaling laws have been generalized for non-uniform heating (with footpoint-concentrated heating functions $E_{H}(h) \propto \exp \left(-h / s_{H}\right)$ characterized by an exponential scale height $s_{H}$ ) and gravitational stratification by Serio et al. (1981). Modeling of the temperature and density profiles of EUV loops observed with TRACE clearly revealed that non-uniform heating functions according to Serio's model fit the data much better than the uniform heating model of Rosner et al. (1978). The results from 41 analyzed loops are shown in Fig. 3, where an average heating scale height of $s_{H}=16.9 \pm 6.1 \mathrm{Mm}$ was found, for loops with a range of loop half lengths $L=4, \ldots, 400 \mathrm{Mm}$ (Aschwanden et al. 2000b). This result applies not only to cool EUV loops, but also to hotter soft X-ray loops observed with Yohkoh (Aschwanden 2002b). These results indicate that coronal heating occurs mainly in the lowest $20 \mathrm{Mm}$ above the solar surface, probably produced by magnetic reconnection processes in the tangled magnetic fields in the complex interface between the chromosphere and corona, called transition region. MHD (DC-braiding) heating simulations (Fig. 4) actually reproduce now the heating scale heights of $s_{H} \approx 20 \mathrm{Mm}$ observed with TRACE (Gudiksen \& Nordlund 2002). These more realistic numerical 3D simulations include the chromospheric structure and transition region, which is a key requirement to reproduce Joule heating concentrated at the footpoints of coronal loops.

\subsection{Magnetic Topology at Footpoints of Coronal Loops}

The localization of the heating function in the footpoint regions of large coronal loops obviously calls for a better understanding of the interface between coronal loops and the chromosphere, the so-called transition region. Conventional hydrostatic models treat the transition region as a very thin layer (with a height extent of a few $100 \mathrm{~km}$ ) in a gravitationally stratified atmosphere. The reality, however, demonstrates that the lowest $5000 \mathrm{~km}$ above the photosphere consist of a very inhomogeneous mixture of cool chromospheric material, warm transition region zones, and hotter coronal plasma, as it could be compellingly observed during the recent Mercury transit, which served as a fitting yardstick of $\approx 5$ $\mathrm{Mm}$. This extended transition region shows a variety of dynamic phenomena, starting from granulation features in photospheric heights (as seen in magnetograms and G-band images), with highly correlated spatial patterns in K-band images (at a height of $\approx 1000 \mathrm{~km}$ ), which merge or bifurcate in a height range of $h \approx 1500-5000 \mathrm{~km}$ in form of spiculae and jets. Hot coronal loops are invisible in the cooler EUV images, but show up as moss features in TRACE $171 \AA$ images where the temperature drops below $T=1.0 \mathrm{MK}$ towards the 

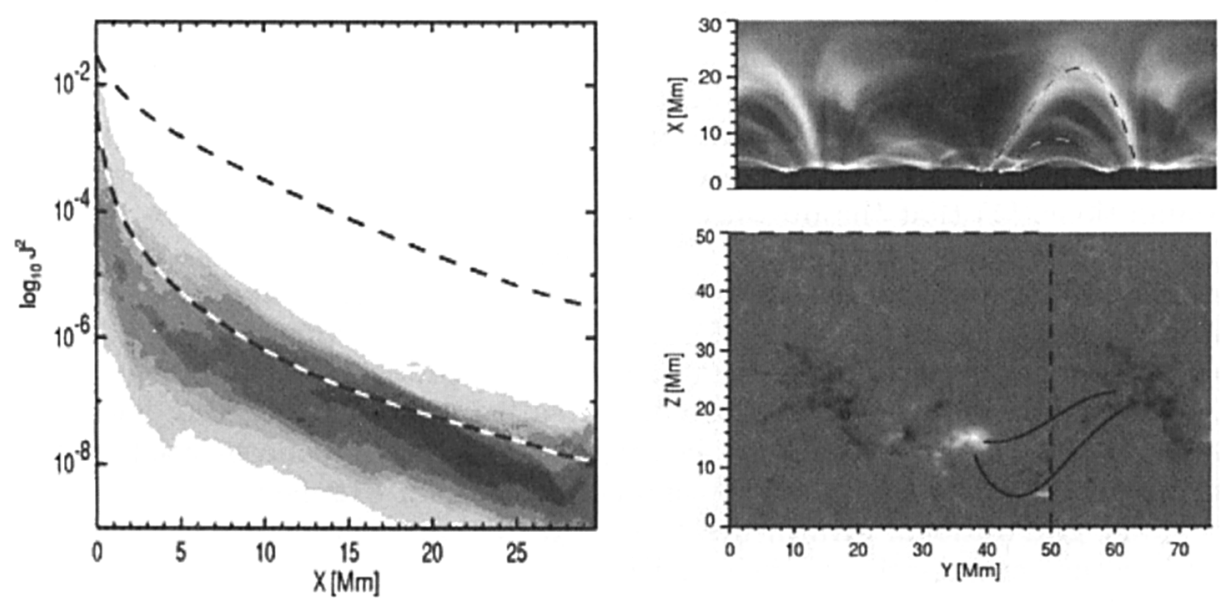

Figure 4. Numerical 3D MHD simulations of coronal heating by DCbraiding. Left: Histogram of current density squared as a function of height, which reproduces the footpoint-concentrated heating observed with TRACE. Right top: Synthetic TRACE 171 A emission measure produced by 3D MHD simulations. Right bottom: SoHO/MDI magnetogram of AR 9114, used as initial condition of the 3D MHD simulations (Gudiksen \& Nordlund 2002).
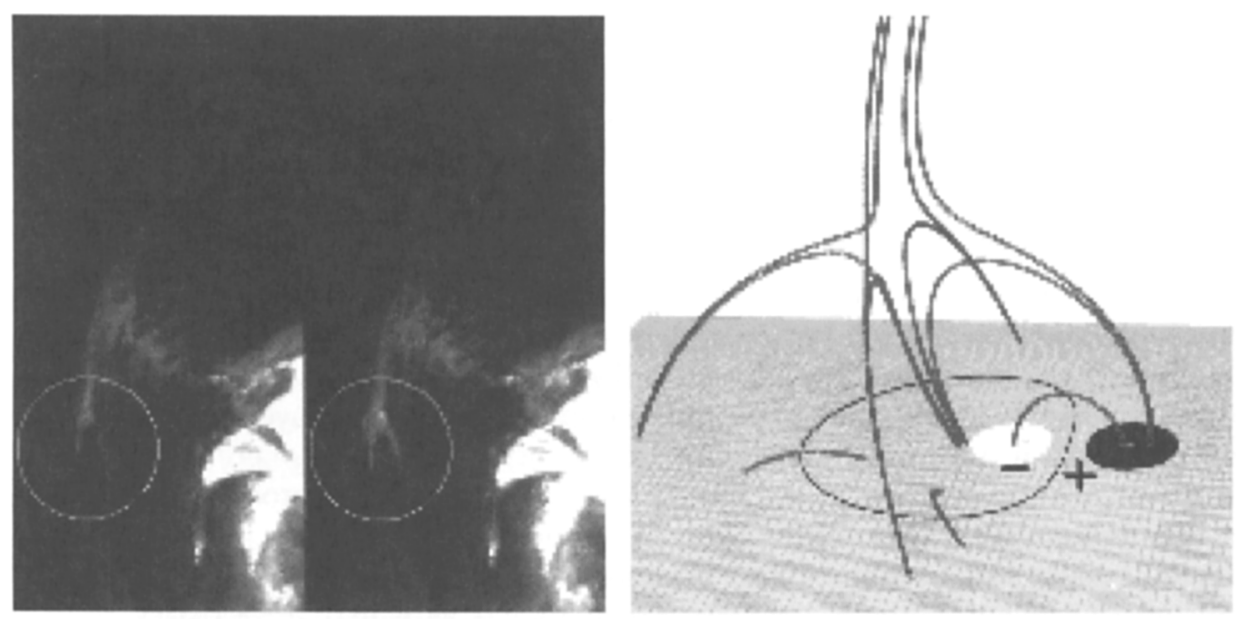

Figure 5. Left: Footpoint detail of a coronal loop recorded with TRACE in $195 \AA$ ( $T \approx 1.5 \mathrm{MK})$. Right: Possible interpretation of the magnetic topology: An isolated magnetic polarity that is surrounded by opposite magnetic polarity forms a dome surface with a 3D magnetic nullpoint (Brown \& Priest 2001; Antiochos 1998). 


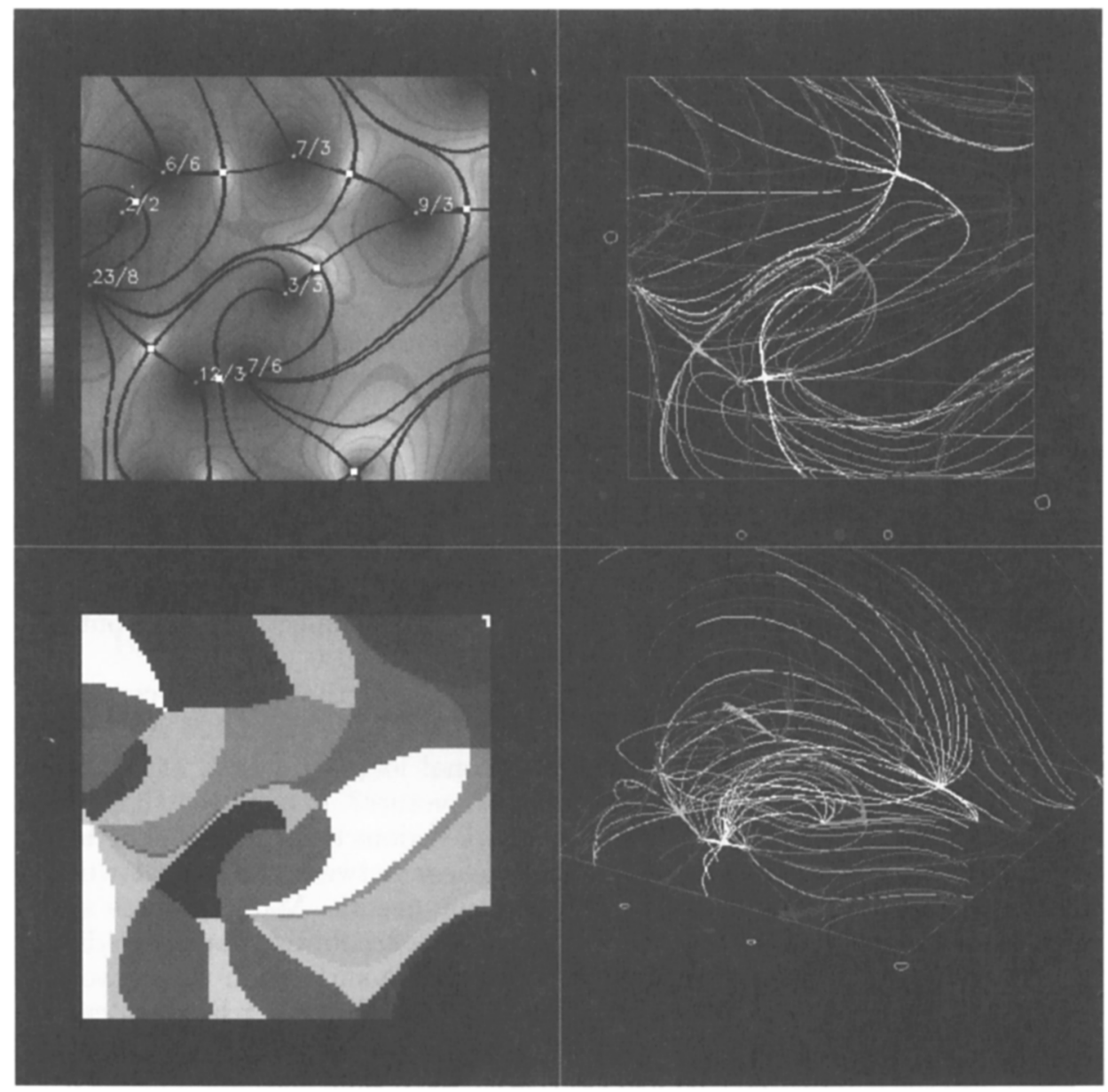

Figure 6. Connectivity domains of a potential magnetic field are visualized by domains with different colors (bottom left). The logarithm of the magnetic field strength is shown with a colored contour map, with nullpoints marked as small white squares and separators are marked with black lines (top left). Fan and spine field lines from different perspectives are shown in the right frames. This numerical computation illustrates that most of the low-lying field lines are closed (in the transition region), while only a small fraction of the field lines are open and connect upward to the corona (Schrijver \& Title 2002). 
chromosphere. The magnetic connectivity from coronal loops is very difficult to track across this messy region, but it appears that coronal loops do not connect directly on the straightest way to the surface (DePontieu, Tarbell, \& Erdélyi 2003), but rather follow along dome-like and canopy-like structures as shown in Fig. 5. Such magnetic topologies have been theoretically modeled in terms of 3D nullpoints, which naturally form in the intersection of a spine field line above an isolated magnetic polarity with a dome-like fan separatrix surface formed by a surrounding circle of opposite magnetic polarity (Brown \& Priest 2001). The same magnetic topology is also found in $\delta$-regions, which are prone to trigger large flares and have been modeled with the so-called magnetic break-out model (Antiochos 1998). At the footpoints of coronal loops, such dome-like separatrix surfaces are thought to form over the size of photospheric granulation cells, supergranulation cells, and network structures, which have typical sizes of $l \approx 1-10 \mathrm{Mm}$. These dome-like separatrix surfaces enclose chromospheric smallscale loops that to not connect upward to the corona, while only a small fraction of field lines can connect upward to coronal loops along the separatrix surfaces. In a recent model it was estimated that $95 \%$ of the photospheric flux closes low down (a tightly weaved texture that is called magnetic carpet), while only the remaining $5 \%$ form large-scale connections upward into the corona (Priest, Heyvaerts, \& Title 2002). This scenario was simulated with detailed magnetic field extrapolations (Schrijver \& Title 2002). An example of these calculations is shown in Fig.6, where magnetic domains of common connectivities are outlined by chromospheric separator lines (Fig. 6, left).

The transition region is highly dynamic. We found that the auto-correlation time of moss structure in C IV $(1550 \AA)$ is $\approx 50 \mathrm{~s}$, in 171 and $195 \AA$ it is only slightly longer with $\approx 70-80 \mathrm{~s}$, and in coronal loop structures at $171 \AA$ we find $\approx 300 \mathrm{~s}$. Which flows drive this dynamic picture? It has been shown that the subphotospheric convection in intranetwork regions transports magnetic flux to the network boundaries, where magnetic shear between colliding downflows is expected to relax by small-scale reconnection events. Many of these smallscale reconnection events heat the chromosphere, accounting for the radiative loss. Reconnection events that occur near separatrix surfaces in the network as well as near open field lines in intranetwork areas that connect between canopy structures up to the corona (Schrijver \& Title 2002) are able to pump heated plasma into the corona and this way contribute to coronal heating. This scenario is also consistent with the footpoint-concentrated heating function inferred from TRACE observations (see $\S 2.3$ ).

\subsection{Coronal Heating Budget}

Since coronal heating appears to be controlled by magnetic reconnection processes in the transition region, we can estimate an upper limit of the available free magnetic energy from the rate of emerging magnetic flux. Of course, the pattern of emerging flux is highly inhomogeneous, but shows the strongest concentrations on spatial scales of $\approx 5 "-10$ " associated with network and mesogranulation boundaries (e.g., Domínguez-Cerdeña, Sánchez-Almeida, \& Kneer 2003). In order to estimate the total rate of emerging magnetic flux, one has to sample the whole size range from small (ephemeral regions) to large features (active regions). Fig. 7 shows a frequency distribution of the emergence rate of 


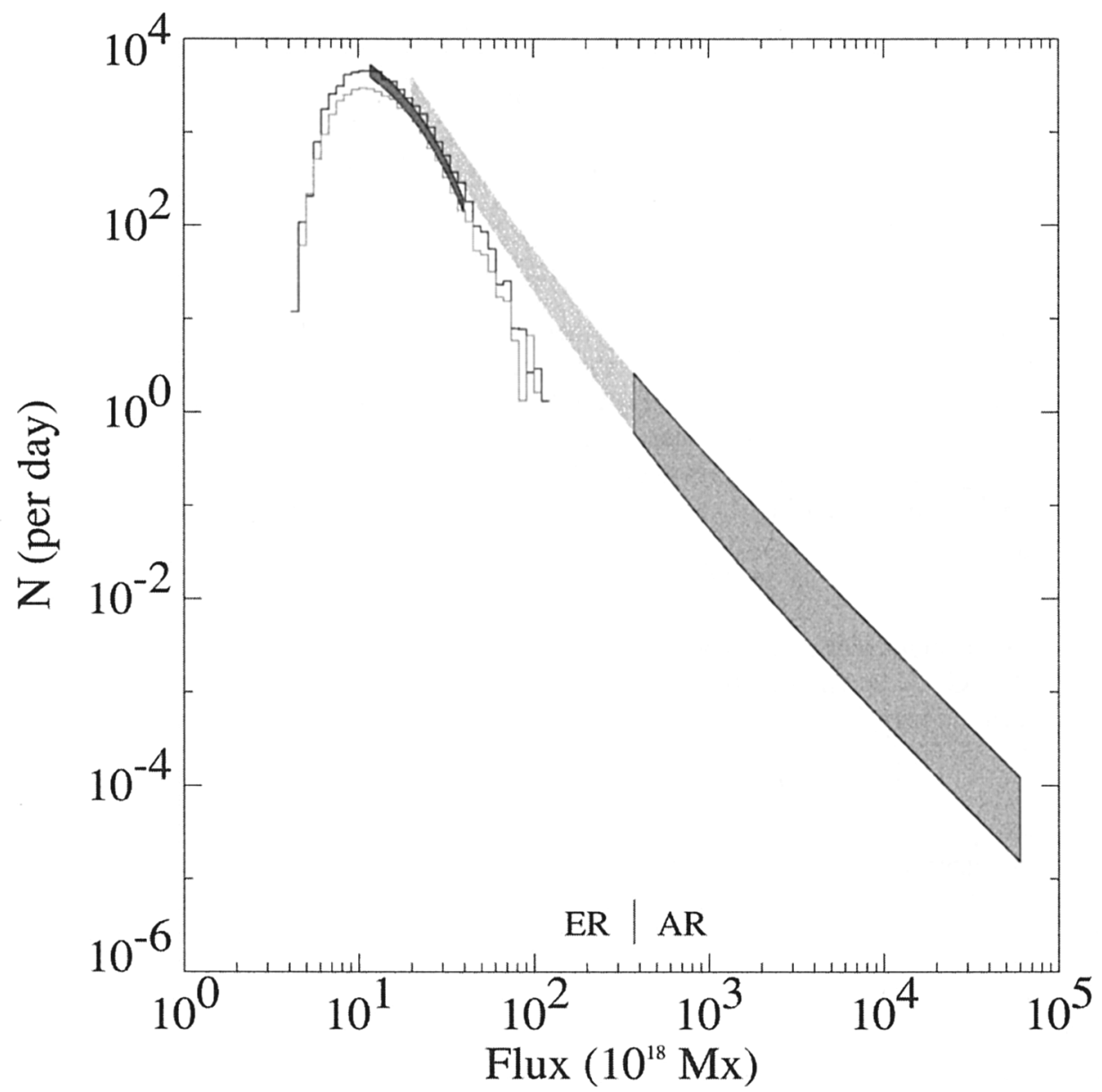

Figure 7. Frequency distribution of emerging magnetic bipoles per day, per flux unit of $\Phi\left[10^{18} \mathrm{Mx}\right]$. The distribution includes ephemeral regions (ER: $\Phi \lesssim 3 \times 10^{20} \mathrm{Mx}$ ) and active regions (AR: $\Phi \gtrsim 3 \times 10^{20}$ $\mathrm{Mx}$, area $A \gtrsim 2.5 \operatorname{deg}^{2}$ ). The variation by a factor 8 is mainly caused by the solar cycle. The histograms include ephemeral regions studied with MDI, with a detection threshold of $\Phi \gtrsim 4 \times 10^{18} \mathrm{Mx}$ (Hagenaar, Schrijver, \& Title 2003). 
magnetic dipoles, which are called ephemeral regions (Harvey \& Martin 1973) if they have an area smaller than $2.5 \mathrm{deg}^{2}$, or active regions if they are larger. The size distribution shown in Fig. 7 encompasses 4 orders of magnitude in magnetic flux $\left(\Phi \approx 5 \times 10^{18} \ldots 5 \times 10^{22} \mathrm{Mx}\right)$ and 8 orders of magnitude variation in the occurrence rate (Hagenaar et al. 2003). A comparison of the magnetic flux emergence rate with the network flux implies an overall mean replacement time of $\approx 8-19 \mathrm{hr}$ in the quiet Sun (Hagenaar et al. 2003).

To estimate the magnetic energy input by subphotospheric convection in an emerging loop, we can use the area of a typical cell as area factor, and the replacement time as rate, which yields the energy dissipation rate $E_{D}\left(\mathrm{erg} \mathrm{cm}^{-2}\right.$ $\left.\mathrm{s}^{-1}\right)$

$$
E_{D}=\frac{B F}{8 \pi^{2} R T}
$$

where $B$ is the magnetic field strength (Gauss), $F$ is the total flux $\left(\mathrm{Mx} \mathrm{cm}^{-2}\right)$, $R$ is the cell radius $(\mathrm{cm})$, and $T$ is the flux replacement time (s). Alternatively, this can be considered as an estimate of the work done by the flow field to move the magnetic field to where it can cancel with an opposite polarity flux element.

For ephemeral regions the average properties are $F=8 \times 10^{18} \mathrm{Mx}, B=1200$ $\mathrm{G}, R=10^{9} \mathrm{~cm}$ (or $10 \mathrm{Mm}$ ), and $T=36,000 \mathrm{~s}$, to the rate of emerging magnetic energy is

$$
E_{D}^{\text {Supergranulation }}=10^{7} \mathrm{erg}^{\mathrm{cm}^{-2} \mathrm{~s}^{-1}} .
$$

For the internetwork fields we have the parameters: $F=1 \times 10^{17} \mathrm{Mx}, B=$ $50-200 \mathrm{G}, R=10^{8} \mathrm{~cm}$ (or $1 \mathrm{Mm}$ ), and $T=3600 \mathrm{~s}$, to the rate of emerging magnetic energy is

$$
E_{D}^{S G-\text { internetwork }}=(0.5-2.2) \times 10^{6} \mathrm{erg} \mathrm{cm}^{-2} \mathrm{~s}^{-1} .
$$

For the granulation scale fields we have the parameters: $F=5 \times 10^{16} \mathrm{Mx}$, $B=1200 \mathrm{G}, R=5 \times 10^{7} \mathrm{~cm}$, and $T=300 \mathrm{~s}$, to the rate of emerging magnetic energy is

$$
E_{D}^{\text {granulation }}=1.6 \times 10^{8} \mathrm{erg} \mathrm{cm}^{-2} \mathrm{~s}^{-1} .
$$

Thus, all three estimates yield a magnetic flux rate that exceeds the coronal heating requirement for coronal holes, $E_{H} \approx 8 \times 10^{5} \mathrm{erg} \mathrm{cm}^{-2} \mathrm{~s}^{-1}$ and the quiet Sun, $E_{H} \approx 3 \times 10^{5} \mathrm{erg} \mathrm{cm}^{-2} \mathrm{~s}^{-1}$ (Withbroe \& Noyes 1977). Thus there is sufficient magnetic energy available to heat the corona.

\subsection{Oscillations and Waves in Coronal Loops}

The first imaging observations of waves and oscillations in coronal loops have been accomplished with TRACE, thanks to the unprecedented contrast, spatial resolution, and fast cadence. It turned out that these newly detected waves and oscillations do not carry sufficient kinetic energy to be significant for coronal heating, but they provide new diagnostic tools for measuring the coronal magnetic field, densities, and possibly the coronal viscosity. There is a rich variety of MHD oscillations modes, generally classified by wave speed: slow-mode MHD waves have a phase speed close to the acoustic speed $\left(c_{s} \approx 150 \mathrm{~km} \mathrm{~s}^{-1}\right.$ for a $T=1.0 \mathrm{MK}$ plasma), and fast-mode MHD waves have phase speeds of Alfvénic 

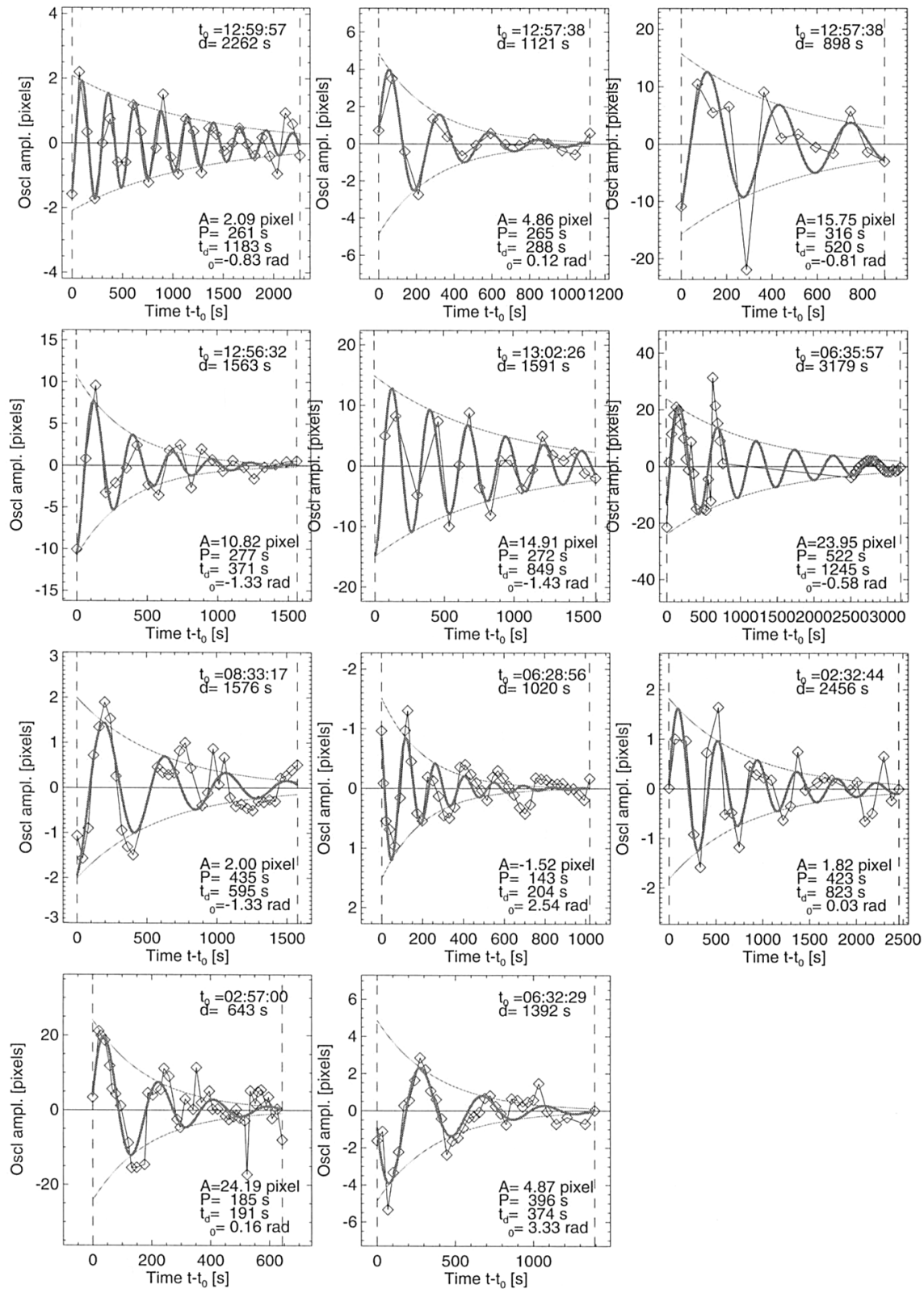

Figure 8. Eleven oscillation events observed with TRACE $171 \AA$, where the transverse MHD kink mode oscillation amplitude $A(t)$ is fitted with a damped sine function plus a low-order polynomial function. The polynomial trend function is subtracted and only the oscillatory fit (thick curve) to the datapoints (diamonds) are shown (adapted from Aschwanden et al. 2002c). 
and magneto-acoustic waves (i.e., typically $v_{A} \approx 1000 \mathrm{~km} \mathrm{~s}^{-1}$. Among the fastmode MHD waves there are symmetric modes (also called "sausage-type") and asymmetric modes (called "kink modes"). The latter type has been discovered with TRACE after flares and filament eruptions, detected by the transverse displacements and the period in the range of $P \approx 3-10 \mathrm{~min}$ that is expected for active region loops (Aschwanden et al. 1999). An unexpected finding was that these MHD oscillations are strongly damped, typically within a few periods (Fig. 8). This strong damping can be explained by two competing mechanisms, either by phase mixing, or by resonant absorption. Both mechanism yield a strong damping consistent with the observations, if the spatial scale of inhomogeneity is on the order of the observed loop radii. However, phase mixing implies also a coronal viscosity that is enhanced by many orders of magnitude over the classical Spitzer value. Besides standing waves of MHD oscillations, propagating waves have also been detected in TRACE loops, which appear to be driven by global 5-minute p-mode oscillations in plages, or 3-minute oscillations in sunspots, respectively (DeMoortel et al. 2002).

\section{Summary}

Reviewing the new findings on coronal loops made with TRACE we learn that the improved instrumental capabilities (spatial resolution, contrast, cadence, temperature discrimination) enable us to measure far more accurate and less contaminated physical parameters than was possible with previous instruments. Thanks to these improved physical parameters we can now probe the highly localized spatial heating function, deviations from hydrostatic equilibria, the energy balance, elemental abundances, magnetic topologies, MHD waves, and oscillations, which all lead to a more rigorous testing of physical models. It is hoped that the improved physical models of the workings in our solar corona can also be transferred and scaled to stellar coronae to some degree.

\section{References}

Antiochos, S.K. 1998, ApJ, 502, L181.

Aschwanden, M.J., Fletcher, L., Schrijver, C., et al. 1999, ApJ, 520, 880.

Aschwanden, M.J., Alexander, D., Hurlburt, N., et al. 2000a, ApJ, 531, 1129.

Aschwanden, M.J., Nightingale, R.W., \& Alexander, D. 2000b, ApJ, 541, 1059.

Aschwanden, M.J. \& Acton, L.W. 2001, ApJ, 550, 475.

Aschwanden, M.J., Schrijver, C.J., and Alexander, D. 2001, ApJ, 550, 1036.

Aschwanden, M.J. 2002a, ApJ, 580, L79.

Aschwanden, M.J. 2002b, COSPAR Coll.Ser.13, (eds. Martens, P. \& Cauffman, D.), 57 .

Aschwanden, M.J., DePontieu, B., Schrijver, C.J., Title, A.M. 2002c, Solar Phys., 206, 99.

Brown, D.S. \& Priest, E.R. 2001, AA, 367, 339.

DeMoortel, I., Ireland, J., Hood, A.W., \& Walsh, R.W. 2002, AA, 387, L13.

DePontieu, B., Tarbell, T., \& Erdélyi, R. 2003, ApJ, 590, 502.

Domínguez-Cerdeña, I., Kneer, F., \& Sánchez-Almeida,J. 2003, ApJ, 582, L55. 
Gudiksen, B.V. \& Nordlund, Å. 2002, ApJ, 572, L113.

Hagenaar, H.J., Schrijver, C. J., \& Title, A.M. 2003, ApJ, 584, 1107.

Harvey, K.L. \& Martin, S.F. 1973, Solar Phys. 32, 389.

Lenz, D.D., DeLuca, E.E., Golub, L., Rosner, R., \& Bookbinder, J.A. 1999, ApJ, 517, L155.

Martens, P.C.H., Cirtain,J.W., and Schmelz, J.T. 2002, ApJ, 577, L115.

Priest, E.R., Heyvaerts, J.F., \& Title, A.M. 2002, ApJ, 576, 533.

Rosner, R., Tucker, W.H., \& Vaiana, G.S. 1978, ApJ, 220, 643.

Schmelz, J.T., Scopes, R.T., Cirtain, J.W., Winter, H.D., \& Allen, J.D. 2001, ApJ, 556, 896.

Schrijver, C.J. and 16 co-authors 1999, Solar Phys., 187, 261.

Schrijver, C.J. \& Title, A.M. 2002, Solar Phys. 207, 223.

Serio, S., Peres, G., Vaiana, G.S., Golub, L., and Rosner, R. 1981, ApJ, 243, 288

Warren, H.P., Winebarger, A.R., \& Hamilton, P.S. 2002, ApJ, 579, L41.

Winebarger, A.R., Warren, H.P., \& Seaton, D.B. 2003, ApJ, 593, 1164.

Withbroe, G.L. \& Noyes, R.W. 1977, Ann. Rev. Astron. Astrophysics, 15, 363. 

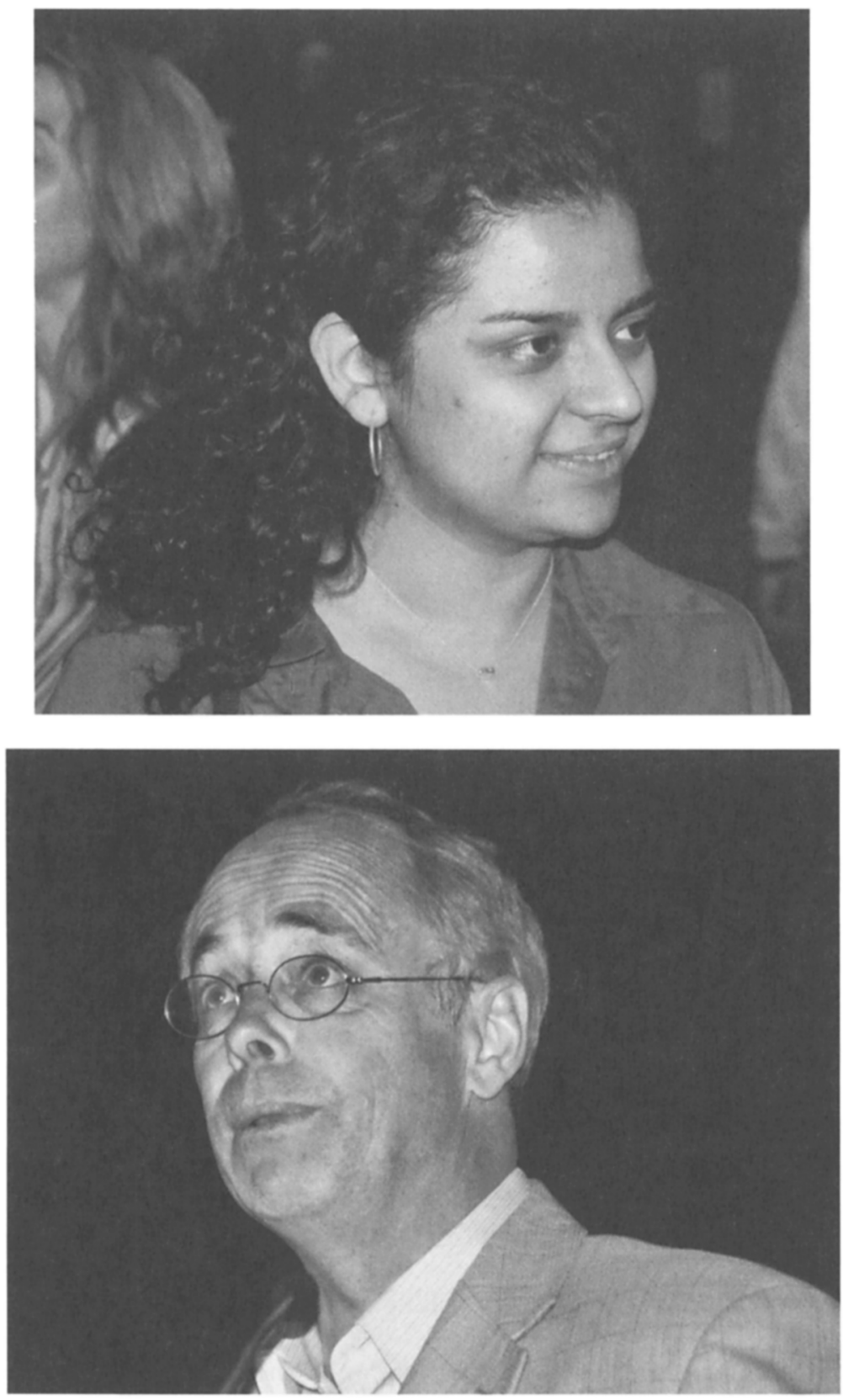